\section{Long-term benefits of chest compression-only cardiopulmonary resuscitation training using real-time visual feedback manikins: a randomized simulation study}

\author{
Tae Chang Jang' ${ }^{1}$, Hyun Wook Ryoo' ${ }^{2}$ Sungbae Moon ${ }^{2}$, Jae Yun Ahn², \\ Dong Eun Lee ${ }^{2}$, Won Kee Lee ${ }^{3}$, Sang Gyu Kwak', Jung Ho Kim ${ }^{5}$ \\ 'Department of Emergency Medicine, Daegu Catholic University School of Medicine, Daegu, Korea \\ ${ }^{2}$ Department of Emergency Medicine, Kyungpook National University School of Medicine, Daegu, Korea \\ ${ }^{3}$ Department of Medical Informatics, Kyungpook National University School of Medicine, Daegu, Korea \\ ${ }^{4}$ Department of Medical Statistics, Daegu Catholic University School of Medicine, Daegu, Korea \\ ${ }^{5}$ Department of Emergency Medicine, Yeungnam University College of Medicine, Daegu, Korea
}

Objective Cardiopulmonary resuscitation (CPR) education with a feedback device is known to result in better CPR skills compared to one without the feedback device. However, its long-term benefits have not been established. The purpose of this study was to evaluate the long-term CPR skill retention after training using real-time visual manikins in comparison to that of non-feedback manikins.

Methods We recruited 120 general university students who were randomly divided into the real-time feedback group (RTFG) and the non-feedback group. Of them, 95 (RTFG, 48; non-feedback group, 47$)$ attended basic life support and automated external defibrillation training for 1 hour. For comparison of retention of CPR skills, the two groups were evaluated based on 2-minute chest compression performed immediately after training and at 3, 6, and 9 months. The CPR parameters between the two groups were also compared using a generalized linear model.

Results At immediately after training, the performance of RTFG was better in terms of average chest compression depth $(51.9 \pm 1.1$ vs. $45.5 \pm 1.1, p<0.001)$ and a higher percentage of adequate chest compression depth $(51.0 \pm 4.1$ vs. $26.9 \pm 4.2, p<0.001)$. This significant difference was maintained until 6 months after training, but there was no difference at 9 months after training. However, there was no significant difference in the chest compression rate and the correct hand position at any time point.

Conclusion CPR training with a real-time visual feedback manikin improved skill acquisition in chest compression depth, but only until 6 months after the training. It could be a more effective educational method for basic life support training in laypersons.

Keywords Heart arrest; Cardiopulmonary resuscitation; Education; Manikins; Simulation training
elSSN: 2383-4625

Received: 9 March 2020

Revised: 28 April 2020

Accepted: 14 May 2020

Correspondence to: Hyun Wook Ryoo Department of Emergency Medicine, Kyungpook National University School of Medicine, 680 Gukchaebosang-ro, Jung-gu, Daegu 41944, Korea

E-mail: ryoo@knu.ac.kr

ORCID

https://orcid.org/0000-0002-1361-9887



How to cite this article:

Jang TC, Ryoo HW, Moon S, Ahn JY, Lee DE, Lee WK, Kwak SG, Kim JH. Long-term benefits of chest compression-only cardiopulmonary resuscitation training using real-time visual feedback manikins: a randomized simulation study. Clin Exp Emerg Med 2020;7(3):206-212. https://doi. org/10.15441/ceem.20.022
This is an Open Access article distributed under the terms of the Creative Commons Attribution Non-Commercial License (https:// creativecommons.org/licenses/by-nc/4.0/). 


Capsule
Wummary is already known
$\begin{aligned} & \text { Previous studies have reported some immediate effects of cardiopulmonary resuscitation (CPR) training with feedback } \\ & \text { devices. However, there is little evidence on the effects of these devices on CPR skill acquisition in laypersons and their } \\ & \text { long-term benefits. }\end{aligned}$
$\begin{aligned} & \text { What is new in the current study } \\ & \text { CPR training with a real-time visual feedback manikin improved skill acquisition in chest compression depth until } 6 \\ & \text { months after the training. It could be a more effective educational method for basic life support training of laypersons } \\ & \text { than manikins without feedback. }\end{aligned}$

\section{INTRODUCTION}

High-quality chest compressions are essential for improving survival following a cardiac arrest. The chest compression rate, depth, hand position, and a full chest recoil are important factors affecting the overall quality of chest compressions. ${ }^{1}$ There are various kinds of cardiopulmonary resuscitation (CPR) training methods and include both instructor-led and self-learning, with various training materials such as videos, audiovisuals, and online classes. ${ }^{2,3}$ Previously, instructor-led training was the most common method because of limited educational materials. However, selflearning programs with or without hands-on practice have been recently developed and are used as alternatives to instructor-led education. ${ }^{2}$ Among the new tools aimed to improve the quality of CPR, real-time feedback systems have been introduced in the last decade. ${ }^{4-13}$ Many of these systems are able to provide real-time feedback on the CPR performance based on measurements of standard CPR parameters, and previous studies have reported immediate effects of CPR training with feedback devices. ${ }^{4-13}$

In a systematic review, Yeung et al. ${ }^{8}$ supported the use of CPR feedback/prompt devices during CPR training to improve skill acquisition and retention. However, these studies had marked heterogeneities in their populations and the types of interventions used. Early published papers on feedback training often reported the use of a traditional American Heart Association (AHA) CPR program with a longer training time and complicated evaluation parameters such as those related to responsiveness, chest compression, ventilation, airway, and automated external defibrillation (AED). ${ }^{14,15}$ Further, evidence on the long-term effects of feedback devices is still limited compared to that for the short-term effects. In addition, most previous studies on CPR training evaluated healthcare providers and students, while only a few of them assessed the laypersons because healthcare providers and students are easier to recruit, and these studies are easy to design. In contrast, recruiting laypersons and their long-term follow-ups are relatively difficult.

However, it is more meaningful to measure the benefits of CPR education among laypersons to popularize the technique and save more lives. We hypothesized that there would be long-term benefits of CPR training using real-time visual feedback on CPR skill acquisition and retention. Accordingly, this study aimed to investigate the long-term benefits of chest compression-only CPR (COCPR) using real-time feedback manikins in comparison to that of non-feedback manikins in laypersons. The primary outcome measure was the mean chest compression depth, whereas the secondary outcome measures were the percentage of adequate chest compression depth and chest compression rate minute.

\section{METHODS}

\section{Study design and participants}

This was a prospective randomized simulation study of general university students. We advertised the recruitment of participants and received applications on a first-come, first served basis without sex restrictions through google survey. The inclusion criteria were age 19 years and university students. There were no exclusion criteria. After stratifying the participants based on sex and prior CPR education, they were randomly assigned to two groups based on the CPR training manikin used as the real-time feedback group (RTFG) and the non-feedback group (NFG). Randomization was achieved via simple block randomization using a random numbering. All participants attended basic life support (BLS) and AED training program and answered a questionnaire survey on age, height, weight, sex, body mass index (BMI), and prior CPR education.

This study was approved by the institutional review board (KNUH 2017-05-005) and was conducted according to the tenets of the Declaration of Helsinki and its seventh revision in 2013. All participants provided a signed consent form. 


\section{Sample size calculation}

The sample size calculation was based on the primary end point (rate of correct compression depth $>80 \%$ ) for comparison of CPR quality between the two groups. The values for the reference were obtained from the report by Skorning et al. ${ }^{9}$ Based on alpha $=0.05$ and a power of $80 \%$, a sample size of 45 per group was needed. Considering a $25 \%$ drop rate, the final sample size was calculated to be 60 participants in each group. In total, 120 participants were included in the study. Of them, 95 (RTFG, 48; NFG, 47) attended the training (Fig. 1).

\section{Blinding and similarity of intervention}

The participants attended training without knowing whether they were divided into groups in advance. However, blinding of instructors was not applied because it was hard to mask using feedback device. To reduce bias, we maximized similarity of education, and all CPR parameters were evaluated and corrected objectively from the CPR simulation manikin. To minimize the educational differences, (1) both groups used the same official CPR program; (2) the instructor could provide feedback, but to minimize intervention by the instructor, the lecture was delivered using a PracticeWhile-Watching method; (3) two certified BLS instructors from the AHA lectured alternately to the RTFG and NFG groups, with each session attended by about 20 students; and (4) both groups used the same CPR training manikin, but one group turned on the feedback system, while the other just turned it off.

\section{Training}

The official BLS and AED training programs from the Korean Association of Cardiopulmonary Resuscitation were employed to train both groups for 1 hour each. The CPR course comprised theory and practice classes that included performing a 2-minute COCPR, four times. The CPR training proceeded in a kneeling position on the hard floor. The ratio of attendees:manikin was 2:1, while the ratio of instructor:attendees was 1:20. We then evaluated their CPR performance by testing their 2-minute COCPR skill immediately after the training in August 2017. To evaluate skill retention, the groups were re-evaluated 3 (November 2017), 6 (February 2018), and 9 months (May 2018) after the training. No further feedback was provided after initial training.

\section{Manikin}

A specific CPR training manikin (BT-SEEM; BT Inc., Goyang, Korea) was used for the study. This manikin had a graphic interface that provided real-time visual feedback of the compression speed and depth. However, it could not provide feedback on the hand position and chest recoil because it is only suitable for training purposes. The tested CPR training simulator was a BT-CPTA, which was created by the same company (BT Inc.).

\section{Study variables}

Information regarding the general characteristics of the participants was collected from the survey. Further, the CPR performanc-

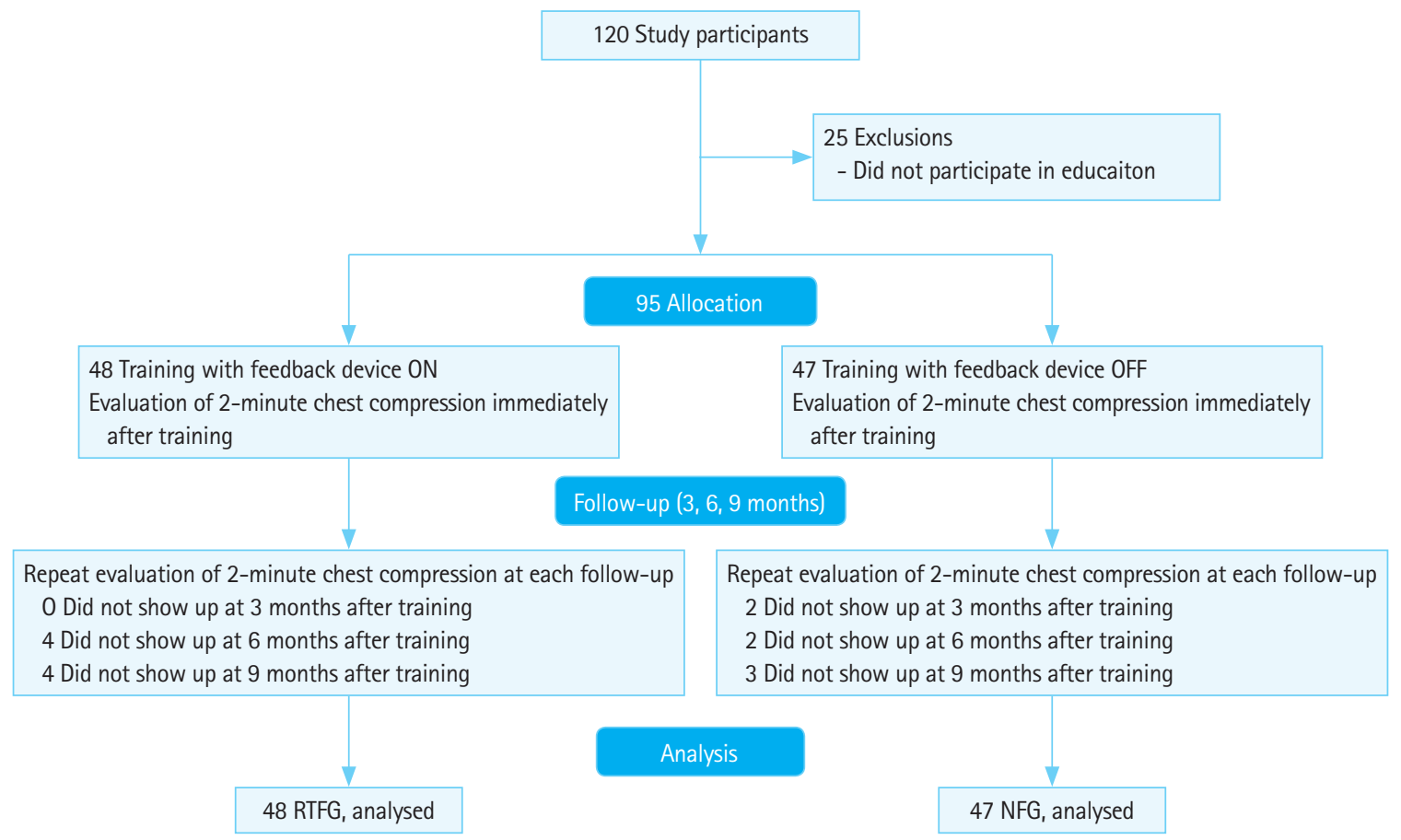

Fig. 1. Flow chart of the study design and participant recruitment. RTFG, real-time feedback group; NFG, non-feedback group. 
es were evaluated using a CPR training simulator. Data on CPR parameters such as chest compression rate, the average depth of chest compression, the percentage of adequate chest compression depth, correct hand position (\%), and chest recoil (\%) were all collected from the CPR training simulator. Adequate chest compression was based on the 2015 guidelines, which included 5-6 $\mathrm{cm}$ chest compression depth and 100-120 per minute chest compression rate. ${ }^{1}$

\section{Statistical analysis}

The general characteristics of the study participants were described as a number (percentage) or median (interquartile ranges). The chi-square test or Fisher exact test were used for comparing proportions. Non-normally distributed continuous variables were analyzed using a non-parametric test (Mann-Whitney test). Betweengroup comparisons of the CPR parameters were performed using the Student t-test at each follow up and a generalized linear model for the overall period. Kolmogorov-Smirnov test method was used for normality testing. All statistical analyses were performed using the IBM SPSS Statistics ver. 19.0 (IBM Corp., Armonk, NY, USA). A P-value $<0.05$ was considered statistically significant.

\section{RESULTS}

We analyzed all participants who underwent training to compare the two groups base on which they were originally allocated (intention-to-treat analysis). There were no significant differences in the general characteristics of the participants, including age, height, weight, BMI, and prior CPR education between the two groups (Table 1). The RTFG performed significantly deeper chest compressions $(P<0.001)$, with a higher percentage of adequate chest compression depth $(P<0.001)$, and significantly lower full chest recoil rate $(P=0.024)$ (Table 2). Other $C P R$ parameters such as the chest compression rate per minute and non-feedback parameters such as the hand position were not significantly different between the two groups (Table 2). The RTFG continued to perform significantly deeper chest compressions $(\mathrm{mm})$ until 6 months after training (51.9 vs. 45.5 after training $[P<0.001], 50.2$ vs. 44.6 at 3 months $[P<0.001]$, and 48.4 vs. 44.8 at 6 months $[P=0.029])$ (Fig. 2). They also achieved adequate chest compression depth rate (\%) until 6 months after training (51.0 vs. 26.9 after training $[P<0.001], 42.3$ vs. 22.9 at 3 months $[P=0.002]$, and 31.8 vs. 22.5 at 6 months $[P=0.041])$ (Fig. 3). However, at 9 months after the

Table 1. General characteristics of the participants $(n=95)$

\begin{tabular}{lccc}
\hline & RTFG $(\mathrm{n}=48)$ & NFG $(\mathrm{n}=47)$ & P-value \\
\hline Age $(\mathrm{yr})$ & $21.0(20.0-22.0)$ & $21.0(20.0-22.0)$ & 0.268 \\
Height $(\mathrm{cm})$ & $163(158-170)$ & $162(156-168)$ & 0.156 \\
Weight $(\mathrm{kg})$ & $52.0(50.3-64.5)$ & $52.0(48.0-60.0)$ & 0.142 \\
Sex, male & $10(20.8)$ & $9(19.1)$ & 0.521 \\
Body mass index $\left(\mathrm{kg} / \mathrm{m}^{2}\right)$ & $20.4(19.3-22.7)$ & $20.3(19.0-21.7)$ & 0.320 \\
Prior CPR education & $16(33.3)$ & $15(31.9)$ & 0.529 \\
\hline
\end{tabular}

Values are presented as median (interquartile ranges) or number (\%). RTFG, real-time feedback group; NFG, non-feedback group; CPR, cardiopulmonary resuscitation.

Table 2. Comparison of the cardiopulmonary resuscitation parameters by time and group

\begin{tabular}{|c|c|c|c|c|c|c|c|}
\hline & Immediately after training & 3 Months & 6 Months & 9 Months & Group & Time & Group \& time \\
\hline \multicolumn{8}{|c|}{ Feedback parameters } \\
\hline \multicolumn{8}{|c|}{ Compression rate (per minute) } \\
\hline NFG & $122.4 \pm 2.31$ & $114.7 \pm 2.36$ & $117.8 \pm 2.36$ & $116.2 \pm 2.39$ & \multirow[t]{2}{*}{0.072} & \multirow[t]{2}{*}{$<0.001$} & \multirow[t]{2}{*}{0.919} \\
\hline RTFG & $120.3 \pm 2.29$ & $110.1 \pm 2.29$ & $114.3 \pm 2.39$ & $114.6 \pm 2.39$ & & & \\
\hline \multicolumn{8}{|c|}{ Average chest compression depth (mm) } \\
\hline NFG & $45.5 \pm 1.06$ & $44.6 \pm 1.08$ & $44.8 \pm 1.08$ & $45.5 \pm 1.09$ & \multirow[t]{2}{*}{$<0.001$} & \multirow[t]{2}{*}{0.093} & \multirow[t]{2}{*}{0.095} \\
\hline RTFG & $51.9 \pm 1.05$ & $50.2 \pm 1.05$ & $48.4 \pm 1.09$ & $46.9 \pm 1.09$ & & & \\
\hline \multicolumn{8}{|c|}{$\%$ of adequate chest compression depth } \\
\hline NFG & $26.9 \pm 4.15$ & $22.9 \pm 4.24$ & $22.5 \pm 4.24$ & $25.0 \pm 4.29$ & \multirow[t]{2}{*}{$<0.001$} & \multirow[t]{2}{*}{0.015} & \multirow[t]{2}{*}{0.088} \\
\hline RTFG & $51.0 \pm 4.11$ & $42.3 \pm 4.11$ & $31.8 \pm 4.29$ & $30.1 \pm 4.29$ & & & \\
\hline \multicolumn{8}{|c|}{ Non-feedback parameters } \\
\hline \multicolumn{8}{|c|}{ Correct hand position rate } \\
\hline NFG & $43.1 \pm 6.36$ & $35.8 \pm 6.50$ & $38.2 \pm 6.50$ & $43.7 \pm 6.57$ & \multirow[t]{2}{*}{0.759} & \multirow[t]{2}{*}{0.531} & \multirow[t]{2}{*}{0.943} \\
\hline RTFG & $41.3 \pm 6.29$ & $35.5 \pm 6.29$ & $43.7 \pm 6.57$ & $46.0 \pm 6.57$ & & & \\
\hline \multicolumn{8}{|c|}{ Full chest recoil } \\
\hline NFG & $99.2 \pm 1.41$ & $99.9 \pm 1.44$ & $99.7 \pm 1.44$ & $99.9 \pm 1.46$ & \multirow[t]{2}{*}{0.024} & \multirow[t]{2}{*}{0.994} & \multirow[t]{2}{*}{0.994} \\
\hline RTFG & $97.9 \pm 1.39$ & $97.6 \pm 1.39$ & $97.2 \pm 1.46$ & $96.8 \pm 1.46$ & & & \\
\hline
\end{tabular}

Values are presented as mean \pm standard deviations. Statistical significance was tested using a generalized linear model. NFG, non-feedback group; RTFG, real-time visual feedback group. 


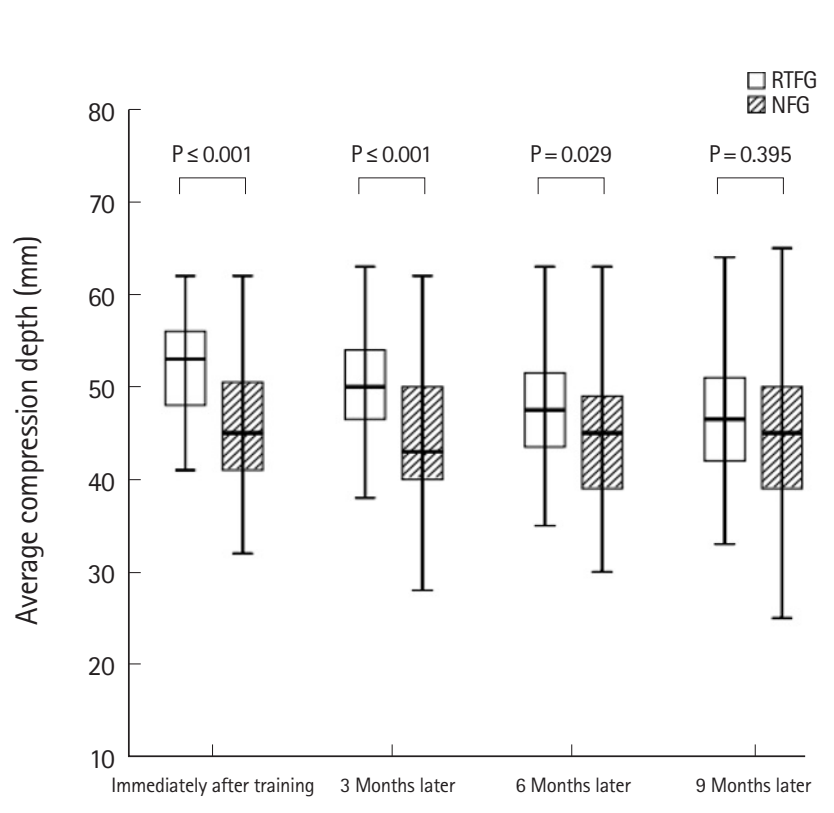

Fig. 2. Comparison of average chest compression depth at each time point of evaluations. Statistical significance was tested using the two sample t-test as a parametric approach. RTFG, real-time feedback group; NFG: non-feedback group.

training, although the RTFG still achieved a higher average compression depth and percentage of adequate chest compression depth, it was no longer significantly different from those of the NFG.

\section{DISCUSSION}

Evidence on the long-term effects of feedback devices for BLS, particularly in laypersons, is still limited. In the current study, we recruited general university students as laypersons, and they participated in a 1-hour BLS/AED training. Compared to the participants who trained with a non-feedback manikin, those who trained with a real-time visual feedback manikin showed higher skill acquisition measured as "adequate chest compression depth." Previous CPR feedback studies in laypersons include one by Wik et al. ${ }^{5}$ that assessed 35 lay volunteers who participated in 20 minutes of training with a computer-based voice advisory manikin system. While training with the feedback system improved the BLS skills, the prompt addition of verbal feedback to the test further improved the performances. Gonzalez-Salvado et al. ${ }^{16}$ studied 155 participants (lay persons and healthcare providers) who received 5 minutes of hands-on instructor-led training along with visual feedback. The laypersons were able to perform overall good quality CPR. In a recently published randomized controlled trial, Baldi et al. ${ }^{17}$ randomly categorized 450 laypersons of various ages

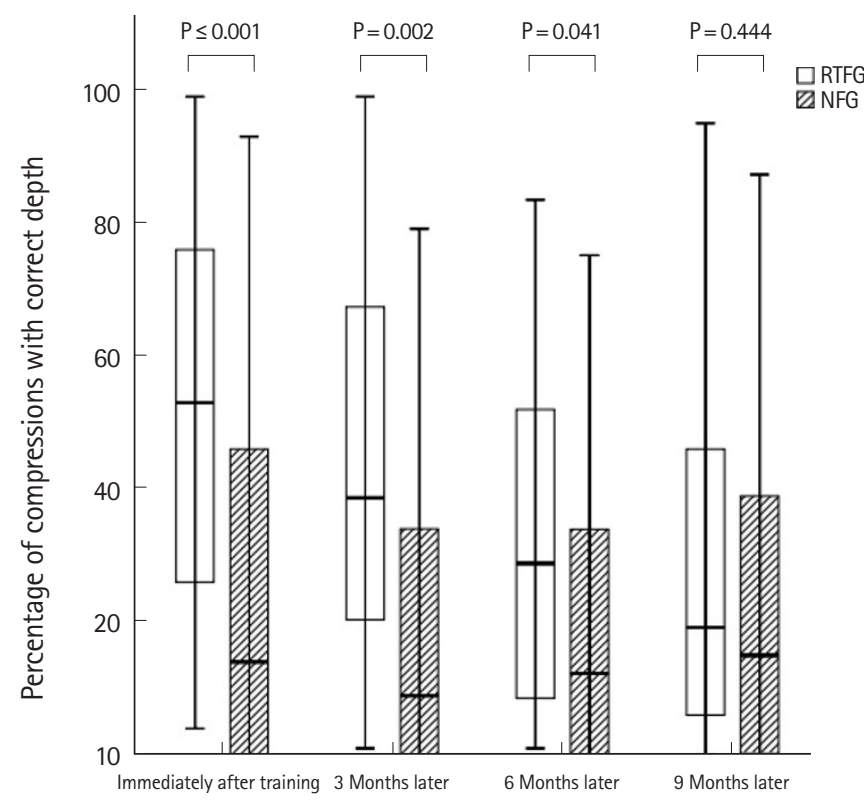

Fig. 3. Comparison of the percentage of adequate chest compression depth at each time point of evaluation. Statistical significance was tested using the two sample t-test as a parametric approach. RTFG, real-time feedback group; NFG: non-feedback group.

participating in BLS/AED courses into three groups. Both 1- and 10-minute training with a feedback device was superior to a course without any feedback with respect to the percentage of compressions with correct depth performed. In another randomized controlled trial, Cortegiani et al. ${ }^{18}$ randomized 144 secondary school students into two arms to compare between a standard course plus a 2-minute chest compression training and a feedback software with an instructor-based training. Using a feedback device resulted in a significantly higher percentage of fully released chest compressions and a better chest compression rate. Collectively, the findings of the present study and these previous studies support the benefits of a feedback system with respect to chest compression skill acquisition in laypersons.

In our study, the CPR parameters were most significantly different between the two groups immediately after the training. The reason for this initial difference might be due to the different quality of feedbacks. We reduced the number of instructors to maximize the dependence and effectiveness of feedback devices, create a situation similar to public education, and minimize instructor interventions during the training. We believe that this initial difference in skill acquisition contributed to the significant differences in the average compression depth and percentage of adequate chest compression depth until 6 months after the training.

The optimal percentage of adequate chest compression in laypersons is hard to define, but it ranged from $58.9 \%$ to $77.8 \%$ with 
a feedback device and from $14.6 \%$ to $66.6 \%$ without a feedback device, respectively in previous studies. ${ }^{17,19}$ Apparently, the rates vary widely and could differ depending on the method of training and participants' characteristics. In our study, the percentage of adequate chest compression depth was $51.0 \%$ and $26.9 \%$ in the RTFG and NFG, respectively. The mean weight and BMI of the participants were $56.4 \mathrm{~kg}$ and $20.4 \mathrm{~kg} / \mathrm{m}^{2}$, respectively. We believe that the relatively lower body weight and mass contributed to the difficulty in performing adequate chest compressions, even with feedback. Oh and $\mathrm{Kim}^{20}$ showed that body weight is highly correlated with chest compression depth in laypersons. Based on their regression equation, rescuers weighing more than $70.5 \mathrm{~kg}$ could achieve a chest compression depth of $50 \mathrm{~mm}$, while lighter rescuers may have difficulty in achieving and maintaining adequate chest compressions. $^{21}$

A recent European Resuscitation Council guideline reported that the CPR skills deteriorate within months of training, and therefore, annual retraining strategies are recommended. However, the optimal intervals for retraining have not been determined and are likely to differ based on the characteristics of the participants. ${ }^{3}$ In our study, skill acquisition after CPR training decreased with time in both groups. In the RTFG, the average chest compression depth was the highest immediately after the training and decreased rapidly with time. Reduction in the accuracy of the CPR performance with time was more rapid in the RTFG than that in NFG. However, the RTFG showed a better mean compression depth and percentage of adequate chest compression depth compared to the NFG until 6 months after the training. These results support the acquisition of better CPR skills through effective training such as that with a feedback system. The reduction in skill retention may, however, be unavoidable with time. Achieving high-quality CPR performance immediately after training could prolong the acquisition of the acquired CPR skills. Wik et al. ${ }^{5}$ reported that their overtraining group, which was provided additional short training sessions with feedback within the next month, had better retention of CPR skills including correct compression depth and inflation. They concluded that effective and qualified training could ensure good CPR performance and long-term skill retention. Even brief but repeated training could improve the CPR skill acquisition and retention. ${ }^{16}$

According to the recent AHA guideline, training methods can be classified based on the baseline skill level of the trainees. For lay rescuers, COCPR is a reasonable alternative to conventional CPR, which is used for adult cardiac arrest patients. On this basis, the Korean Association of Resuscitation also created and distributed CPR training videos of different levels for laypersons (basic/advanced) and for healthcare providers. In our study, we used the ba- sic CPR training program for a layperson, which included lessons on COCPR and the use of AED to investigate the effect of CPR training using a real-time feedback manikin on essential chest compression parameters. A literature review showed that various forms of CPR education yield different results. The results depend on the skill levels of the target participants (layperson or healthcare provider), previous CPR experiences, methods of training (instructor led or self-training), durations of training (very brief to full time), change of training material, and methods of training evaluation (COCPR, conventional CPR, or extended time CPR).4-16 Unlike previous studies, our study was conducted with a specific group of participants (laypersons), educational method (COCPR, Korean CPR training video program), mode of intervention (real-time visual feedback manikin), and parameter assessed (long term skill retention). In the future, if the training method and intervention can be tailored to specific participant groups, the available educational resources can be optimized, and thereby maximize the acquisition of CPR skills immediately after training. This, in turn, will help in extending the long-term benefits of the CPR training.

Our study has a few limitations. First, the study group included university students, which restricts the generalizability of the results to the general population and other age groups. Second, this study is a randomized controlled trial, and we designed each arm to include 60 participants, taking into account potential dropouts. However, $25 \%$ of the participants did not participate in the CPR training, and a few trainees skipped one or more tests. Third, the instructors were not blinded. It would be meaningful to design a study using blinded assessors to reduce bias. Fourth, the CPR training manikin does not give feedback on the correct hand position and complete chest recoil. We believe that the training could be more beneficial if a device that provides feedbacks on multiple CPR parameters is used. Despite these limitations, we believe that our study is valuable because it compares the longterm benefits of CPR training in laypersons between traditional and real-time visual feedback manikins, helping verify the benefits of a real-time feedback manikin before extending this training as a part of public education.

In conclusion, CPR training with a real-time visual feedback manikin improved skill acquisition in chest compression depth metrics, but the advantage disappeared 6 months after the training. It could be a better educational method for BLS training in laypersons comparing with traditional training.

\section{CONFLICT OF INTEREST}

No potential conflict of interest relevant to this article was reported. 


\section{ACKNOWLEDGMENTS}

This study was supported by the Daegu Metropolitan City (number 20161221-0057). The funders had no role in the study design, data collection and analysis, decision to publish, or preparation of the manuscript. We appreciate the Public Health Division of Daegu Metropolitan City Hall for their continued support.

\section{REFERENCES}

1. Kleinman ME, Brennan EE, Goldberger ZD, et al. Part 5: adult basic life support and cardiopulmonary resuscitation quality. 2015 American Heart Association guidelines update for cardiopulmonary resuscitation and emergency cardiovascular care. Circulation 2015;132(18 Suppl 2):S414-35.

2. Hsieh MJ, Bhanji F, Chiang WC, Yang CW, Chien KL, Ma MH. Comparing the effect of self-instruction with that of traditional instruction in basic life support courses: a systematic review. Resuscitation 2016;108:8-19.

3. Greif $R$, Lockey $A S$, Conaghan $P$, et al. European Resuscitation Council Guidelines for Resuscitation 2015: Section 10. Education and implementation of resuscitation. Resuscitation 2015; 95:288-301.

4. Wik L, Thowsen J, Steen PA. An automated voice advisory manikin system for training in basic life support without an instructor. A novel approach to CPR training. Resuscitation 2001;50: 167-72.

5. Wik L, Myklebust $H$, Auestad BH, Steen PA. Retention of basic life support skills 6 months after training with an automated voice advisory manikin system without instructor involvement. Resuscitation 2002;52:273-9.

6. Handley AJ, Handley SA. Improving CPR performance using an audible feedback system suitable for incorporation into an automated external defibrillator. Resuscitation 2003;57:5762.

7. Noordergraaf GJ, Drinkwaard BW, van Berkom PF, et al. The quality of chest compressions by trained personnel: the effect of feedback, via the CPREzy, in a randomized controlled trial using a manikin model. Resuscitation 2006;69:241-52.

8. Yeung J, Meeks R, Edelson D, Gao F, Soar J, Perkins GD. The use of CPR feedback/prompt devices during training and CPR performance: a systematic review. Resuscitation 2009;80:74351.

9. Skorning M, Beckers SK, Brokmann JCh, et al. New visual feedback device improves performance of chest compressions by professionals in simulated cardiac arrest. Resuscitation 2010; 81:53-8.
10. Pozner CN, Almozlino A, Elmer J, Poole S, McNamara D, Barash D. Cardiopulmonary resuscitation feedback improves the quality of chest compression provided by hospital health care professionals. Am J Emerg Med 2011;29:618-25.

11. Kirkbright S, Finn J, Tohira H, Bremner A, Jacobs I, Celenza A. Audiovisual feedback device use by health care professionals during CPR: a systematic review and meta-analysis of randomised and non-randomised trials. Resuscitation 2014;85: 460-71.

12. Beckers SK, Skorning MH, Fries M, et al. CPREzy improves performance of external chest compressions in simulated cardiac arrest. Resuscitation 2007;72:100-7.

13. Spooner BB, Fallaha JF, Kocierz L, Smith CM, Smith SC, Perkins GD. An evaluation of objective feedback in basic life support (BLS) training. Resuscitation 2007;73:417-24.

14. Woollard $M$, Whitfeild $R$, Smith $A$, et al. Skill acquisition and retention in automated external defibrillator (AED) use and CPR by lay responders: a prospective study. Resuscitation 2004; 60:17-28.

15. Einspruch EL, Lynch B, Aufderheide TP, Nichol G, Becker L. Retention of CPR skills learned in a traditional AHA Heartsaver course versus $30-$ min video self-training: a controlled randomized study. Resuscitation 2007;74:476-86.

16. Gonzalez-Salvado V, Fernandez-Mendez F, Barcala-Furelos R, Pena-Gil C, Gonzalez-Juanatey JR, Rodriguez-Nunez A. Very brief training for laypeople in hands-only cardiopulmonary resuscitation. Effect of real-time feedback. Am J Emerg Med 2016;34:993-8.

17. Baldi $E_{1}$ Cornara $S$, Contri $E_{1}$ et al. Real-time visual feedback during training improves laypersons' CPR quality: a randomized controlled manikin study. CJEM 2017;19:480-7.

18. Cortegiani A, Russotto V, Montalto F, et al. Use of a real-time training software (Laerdal QCPR) compared to instructor-based feedback for high-quality chest compressions acquisition in secondary school students: a randomized trial. PLoS One 2017; 12:e0169591.

19. Wutzler $A$, von Ulmenstein $S$, Bannehr $M$, et al. Improvement of lay rescuer chest compressions with a novel audiovisual feedback device : a randomized trial. Med Klin Intensivmed Notfmed 2018;113:124-30.

20. Oh JH, Kim CW. Relationship between chest compression depth and novice rescuer body weight during cardiopulmonary resuscitation. Am J Emerg Med 2016;34:2411-3.

21. Krikscionaitiene $A$, Stasaitis $K$, Dambrauskiene $M$, et al. Can lightweight rescuers adequately perform CPR according to 2010 resuscitation guideline requirements? Emerg Med J 2013; 30:159-60. 